\title{
Study on the Validity of Wien's Displacement Law on Tungsten Bulb
}

\section{R. Neupane}

\section{Journal of Nepal Physical Society}

Volume 6, Issue 2, December 2020

ISSN: 2392-473X (Print), 2738-9537 (Online)

\section{Editors:}

Dr. Binod Adhikari

Dr. Bhawani Joshi

Dr. Manoj Kumar Yadav

Dr. Krishna Rai

Dr. Rajendra Prasad Adhikari

Mr. Kiran Pudasainee

JNPS, 6 (2), 85-96 (2020)

DOI: http://doi.org/10.3126/jnphyssoc.v6i2.34863

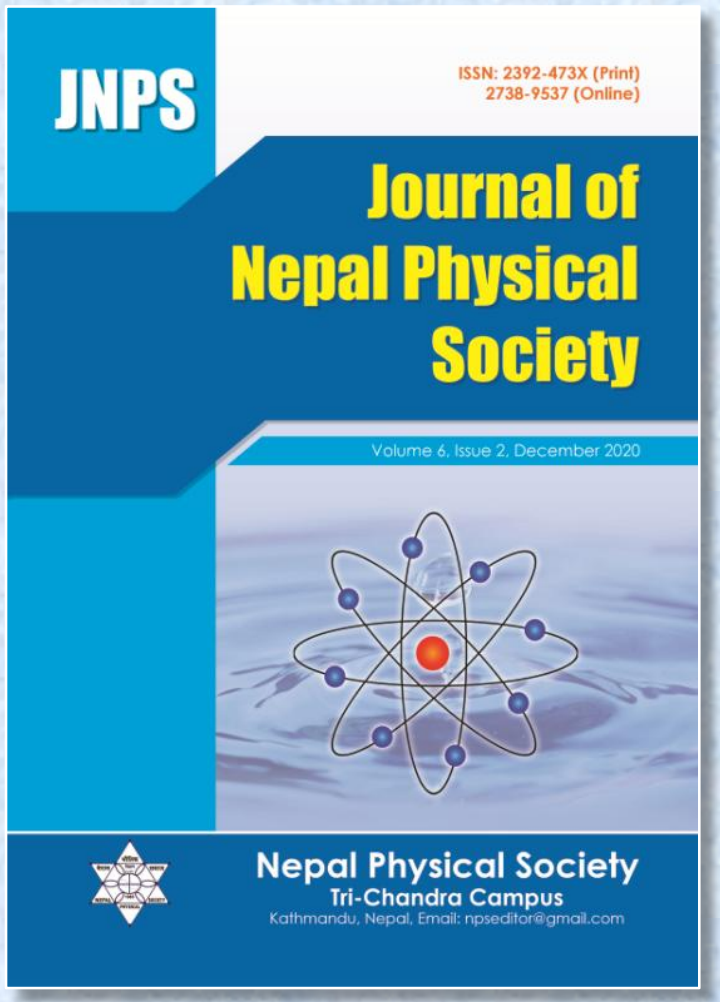

\section{Published by:}

Nepal Physical Society

P.O. Box: 2934

Tri-Chandra Campus

Kathmandu, Nepal

Email: npseditor@gmail.com 


\title{
Study on the Validity of Wien's Displacement Law on Tungsten Bulb
}

\author{
R. Neupane \\ Department of physics, Birendra Multiple Campus, Tribhuvan University, Bharatpur, Nepal \\ Corresponding Email: rajendra.neupane@bimc.tu.edu.np
}

Received: 20 October, 2020; Revised: 30 November, 2020; Accepted: 28 December, 2020

\begin{abstract}
This work was mainly based on three interdependent parameters, which are temperature, emissivity and peak emission wavelength. Temperature is the primary parameter that determines how much light the filament gives off, and at what wavelengths. The work was focused on temperature determination of tungsten filament with different values of emissivity. The different values of emissivity taken for the work were $0.433,0.431,0.427$, 0.421 and 0.415 . Peak emission wavelength was calculated at different tungsten temperatures for different wattage bulbs which were in the order of $10^{-6} \mathrm{~m} .6,60$, and 500 watt bulb were taken for the work. The peak of the spectrum lay in the infrared region. Wien's displacement law was used to calculate the value of peak emission wavelength. The work was based on theoretical model. Blackbody spectrum curve was used to analyze the emitted radiations from the bulb. In each spectrum curve, radiations having higher wavelengths were emitted in greater amount than the radiations having lower wavelength. Spectral radiance was found to be dependent upon both emissivity and power of the bulb. The area under the blackbody spectrum curve indicated the total number of emitted radiations and hence the total energy radiated across all wavelengths. The total energy emitted from tungsten filament was found to be increased rapidly with temperature. Brightness of the bulb increased with the increase in temperature of the tungsten. The peak in the blackbody spectrum curve shifts towards left, when temperature increased. There is a direct consequence of the brightness of bulb with the peak emission wavelength.
\end{abstract}

Keywords: Emissivity, Filament, Peak emission wavelength, Spectrum.

\section{INTRODUCTION}

Tungsten filaments are used in incandescent lamps and radio tubes. They are also used in purely scientific and engineering investigations where high temperature measurements are involved. The melting point of tungsten is 3400 degree Celsius. The high temperatures at which the tungsten filament can be operated render it especially suitable for these purposes [1]. By the late $19^{\text {th }}$ century, scientists found that, bodies would glow when heated, and their color would depend not on their material but on their temperature [2]. The temperature of the glass bulb was found experimentally by Carla Isabel Ribeiro. He connected the lamp to the variable DC energy supply and the temperature was measured with digital thermometer. He found that as the voltage increases, the temperature of the filament and bulb also increases. In his work he obtained different values of temperature of the filament and bulb on varying applied potential [2]. On a single bulb, he found that at a low voltage, the lamp shines a dim red light. As the voltage and the filament temperature increased, the light became more intense and changed the colour [2]. He used 3.5 volt light bulb and a variable DC. He used a single bulb at varying potential.

In this work, I have observed the spectrum of possible radiations emitted from tungsten bulb at constant supply of voltage. This work show, Wien's displacement law works well for a body heating at temperature range from $2015 \mathrm{~K}$ to $2730 \mathrm{~K}$. I have explained this on the basis of the spectrum of the radiations emitted from the tungsten bulb. I have used tungsten as a blackbody to check the validity of Wien's displacement law on it. Light of an 
accurately defined color and brilliancy could be found by heating a tungsten filament to a particular temperature. The intensity and color of the light could be explained by the Stefan - Boltzmann and Wien's displacement laws. As the temperature raised, the body glow with the colors corresponding to even- smaller wavelengths of the electromagnetic spectrum. This is the underlying principle of the incandescent light bulb [3]. A black body doesn't exist and is theoretical [3]. The spectrum curve of the blackbody radiation was shown by Yang and Wei. The curve was plotted for the blackbodies having temperature range from $3500 \mathrm{~K}$ to $5500 \mathrm{~K}$ [4].

In this work, I have shown simulated blackbody radiation curve for temperature range of $2015 \mathrm{~K}$ to 2730 K. Different wavelength radiations from the tungsten filament gives rise to an electromagnetic spectrum which is discussed in section 1.1.

\subsection{Electromagnetic Spectrum}

The electromagnetic spectrum was explored in the year 1800, when Sir William Herchel's discovered "obscure rays" extending beyond the red end of the visible spectrum [5]. In the following year Johann Ritter demonstrated that invisible rays beyond the violet end of the spectrum are capable of chemical action. These three adjacent portions of the electromagnetic spectrum: infrared, visible and ultraviolet are collectively known as optical radiation. There are no sharp, well- defined boundaries in the electromagnetic spectrum. The different radiations with their wavelength range were described by David et al. which are shown in Table 1 [5]. Electromagnetic radiation is a form of energy that is all around us and takes many forms, such as radio waves, microwaves, $\mathrm{x}$-rays and gamma rays, etc. Electromagnetic spectrum explains how the electromagnetic waves are divided into different ranges, depending on their wavelength and frequency [6].

Visible light is a form of electromagnetic radiation, which can be perceived by our eyes. The wavelength of visible radiations (Light) varies from $380 \mathrm{~nm}$ to $740 \mathrm{~nm}$. Various spectral color bands make up light. The band from 100 $\mathrm{nm}$ to $400 \mathrm{~nm}$, is shown in Table 1 is called ultraviolet radiation. The band from $710 \mathrm{~nm}$ to 1.5 micrometers is called near infrared and the band from 1.5 to 4.0 micrometers is called far infrared [7]. The wavelength ranges of different colors of visible light shown in Table 2 were used by Andrew Zimmerman to explain the emitted radiation from the heating body [8].
Table 1: Radiations with their wavelength \{Ref: [5]\}

\begin{tabular}{ll}
\hline Ultraviolet & $100-400 \mathrm{~nm}$ \\
\hline Light & $380-400$ to $760-780 \mathrm{~nm}$ \\
Infrared & $760-780 \mathrm{~nm}$ to $1 \mathrm{~mm}$ \\
\hline
\end{tabular}

Table 2: The Visible light spectrum \{Ref: [8]\}

\begin{tabular}{ll}
\hline Color & Wavelength(nm) \\
\hline Red & $625-740$ \\
Orange & $590-625$ \\
Yellow & $565-590$ \\
Green & $520-565$ \\
Cyan & $500-520$ \\
Blue & $435-500$ \\
Violet & $380-435$ \\
\hline
\end{tabular}

\section{LITERATURE REVIEW}

A blackbody can be described as an enclosed body which absorbs all the incident radiations upon it. When the blackbody is at equilibrium, it will emit radiation at the same rate as it absorbs radiation from the surrounding medium. Sometimes a blackbody is referred to as a complete absorber [9]. The temperature and wavelength relation, and temperature and emissivity relation is described in section 2.1 and 2.2 respectively.

\subsection{Wavelength And Temperature}

In Wien's displacement law, source temperature and wavelength are related. Wien's displacement equation states that the product of the peak wavelength and the source temperature is constant. This implies that the peak of the radiation shifts to shorter wavelengths when the temperature increases [10]. Wien's displacement law holds for shorter wavelengths where as Rayleigh's Jein's law for longer wavelength [11]. Wien's displacement law has an important part in the development of modern quantum theory, and predicting it was one of the triumphs of Planck's distribution law [12].

\subsection{Temperature And Emissivity}

The emissivity of tungsten wire filaments in incandescent lamps changed with wavelength. The lamp showed better performance if the emissivity of the filament was higher [13]. The emissivity $\varepsilon$ at a given wavelength $\lambda$ and temperature $T$, is defined as the ratio of the 
radiance emitted by a body at temperature $T$ and the radiance emitted by a black body at the same temperature $T$ [14]. The emissivity had been increased on increasing temperature [15]. The emissivity and temperature profile is shown in Table 3.

Table 3: Variation in spectral emissivity with temperature $\{$ Ref: [15]\}

\begin{tabular}{ll}
\hline \multicolumn{1}{c}{$\begin{array}{c}\text { Temperature in } \\
\text { Kelvin }(\boldsymbol{T})\end{array}$} & $\begin{array}{c}\text { Spectral } \\
\text { Emissivity }(\boldsymbol{\varepsilon})\end{array}$ \\
\hline 1900 & 0.437 \\
2000 & 0.435 \\
2100 & 0.433 \\
2200 & 0.431 \\
2300 & 0.429 \\
2400 & 0.427 \\
2500 & 0.425 \\
2600 & 0.423 \\
2700 & 0.421 \\
2800 & 0.419 \\
2900 & 0.417 \\
3000 & 0.415 \\
3100 & 0.413 \\
\hline
\end{tabular}

The energy emitted per unit area per unit time depends upon the emissivity of the material. The total emissivity can be calculated by using the Equation 1:

$$
P=8 \sigma \mathrm{A} T^{4}
$$

Where $\varepsilon$ is the total emissivity, $\sigma$ is Stefan's Constant, $A$ is the area of the tungsten, $T$ is the temperature [15].

\subsection{Stefan's Boltzmann Constant}

The Stefan-Boltzmann constant $\sigma$ had been evaluated by using an absolute radiometer of the electrical substitution type to measure the radiance of a cavity radiator at the freezing point of gold. The value obtained for $\sigma$ was $(5.6644 \pm 0.0075) \mathrm{x}$ $10^{-8} \mathrm{~W} \mathrm{~m}^{-2} \mathrm{~K}^{-4}[16]$.

\subsection{Resistivity and Temperature}

The value of resistivity goes on increasing with increase in temperature. The different values of resistivity with temperature are shown in Table 4.
Table 4: Value of resistivity of the filament with temperature \{Ref: [17]\}

\begin{tabular}{ll}
\hline Temperature $(\mathbf{K})$ & Resistivity $(\boldsymbol{\mu} \boldsymbol{\Omega} . \mathbf{c m})$ \\
\hline 2200 & 63.48 \\
2300 & 66.91 \\
2400 & 70.39 \\
2500 & 73.91 \\
2600 & 77.49 \\
2700 & 81.04 \\
\hline
\end{tabular}

\section{MATERIALS AND METHODS}

Six filament bulbs of $6,25,60,100,300,500$ watt are taken. The values of length and diameter of these filament bulbs had been measured by Agrawal D.C. [18]. I have used these verified data for my work. In this paper, for same wattage heating bulb, different values of temperature of the heating filament have been calculated at different values of emissivity. The values of emissivity vary from 0.433 to 0.413 for filament temperatures from $2100 \mathrm{~K}$ to $3100 \mathrm{~K}$ respectively as shown in Table 3.The spectrum has been analyzed at many different wavelengths with the help of simulated blackbody emission curve. The value of resistivity is taken $7.391 \times 10^{-5} \Omega \mathrm{cm}$ assuming the mean temperature of the filament rises to $2500 \mathrm{~K}$ as shown in Table 4 . This value of resistivity is kept constant for each wattage bulb. The values of emissivity for each wattage bulb are taken from 0.433 to 0.415 which is shown in Table 4 . In peak emission wavelength, the three digits after the decimal are also included. The third decimal digit is rounded to nearer value. The lower limit and upper limit of wavelength, has been taken as $2.7 \times 10^{-7} \mathrm{~m}$ and $6 \times 10^{-6} \mathrm{~m}$ respectively. These extreme values of wavelength are chosen considering, the distribution of the emitted radiations from the tungsten bulb are probabilistic and the wavelength of the emitted radiations will lie within this wavelength range. The power of the tungsten bulb has been calculated in terms of voltage, radius of the tungsten wire, its length and resistivity. The temperature of the filament bulb is calculated by using Equation 8. The peak value of the wavelength at different temperatures has been calculated using Wien's displacement law in Equation 9. Physical quantities like spectral radiance, radiant emittance, peak spectral radiance and band radiance have been calculated by using blackbody spectral calculator [19]. Six different figures of blackbody spectrum 
curve have been drawn for different wattage bulb which operates on different temperatures.

The modality is based on the assumption that "the filament lamp distributes more amounts of infrared radiations and very few amounts of ultraviolet radiations. The visible radiations lie in intermediate level between lower and higher wavelength limit of the emitted radiations". The filament lamp distributes spectrum of wavelength of emitted radiations. The different figures made with different values of emissivity show "how the spectrum of the wavelength of the emitted radiations is distributed". The different figures at different temperatures, between spectral emittance and wavelength are plotted with the help of $\mathrm{Hi}$-resolution spectral calculator [19]. The wavelength of the emitted radiations obtained in spectrum curve from Fig.1-6 has been selected with the help of the spectrum Table 1-2. The key equations used for this work are temperature determination equation and peak emission wavelength determination equation which are given in Equation 8 and 9 respectively. The temperature determination equation is illustrated in section 3.1 .

\subsection{Temperature Calculation Methods}

Temperature of the heating filament has been measured theoretically in terms of its length, radius and resistivity. Since voltage is a constant in our electrical distribution system, it is taken same to calculate the temperature of the heating filament for each wattage bulb.

When a light bulb is turned on using a switch, a constant (ac) voltage $V$ of $120 \mathrm{~V}$ is applied across the tungsten filament. Since the tungsten filament has a high value of resistance, because of its fine diameter and long length (see Equation(5)), a small amount of current flows through the filament in accordance with Ohm's law. The Ohm's law is given in Equation 2.

$$
V=I R
$$

Where, $I$ is current, $V$ is voltage across the filament and $R$ is resistance of the filament [20]. The tungsten filament then becomes hot, since amount of power $P$ produced in the filament is

$$
P=\frac{V^{2}}{R}
$$

Since voltage is a constant, the form of equation for the power dissipated in a resistor should be that shown in equation 3 , and should not be $P=I^{2} R$ .Since $V$ is a constant, Equation (3) relates one variable $R$ to another variable $P$. If the equation
$P=I^{2} R$ was used, then $P, I$ and $R$ would all be variables. The filament is compared to a theoretical blackbody radiator. The total power emitted per unit surface area ${ }_{A}$ ) of a hot object at temperature $T$ (in Kelvin) is given by the Stefan-Boltzmann law [16]:

$$
\frac{P}{A}=\varepsilon \sigma T^{4}
$$

Where, $\sigma$ is known as Stefan- Boltzmann constant, and has the value $5.67 \times 10^{-8} \mathrm{~W} /\left(\mathrm{m}^{2}-\mathrm{K}^{4}\right)$ [16]. The emissivity $\varepsilon$ is a material dependent quantity. For a long cylindrical filament of radius $r$ and length $L$, the cross sectional area $A$ is $\pi r^{2}$ and the surface area $S$ is $2 \pi r l$. Electrical resistance $R$ of material is given by

$$
R=\frac{\rho L}{A}
$$

Combining equations (3) and (5), the power dissipated in the filament can be written as

$$
P=\frac{V^{2} \pi r^{2}}{\rho L}
$$

Since voltage in our houses is fixed, it is apparent from Equation (3) that for higher wattage bulbs, electrical resistance of the filament must decrease as bulb wattage increases. From Equation (5), $R$ can be decreased either by increasing $A$ (i.e., $r^{2}$ ) or by decreasing $L$. Therefore, for higher wattage bulbs, it is necessary to either increase $r$ or decrease $L$.From the Stefan- Boltzmann law [21], the power emitted by the tungsten filament can also be written as

$$
P=\varepsilon \sigma T^{4}(2 \pi r L)
$$

From this equation, we can see that the bulb wattage (brightness), filament temperature (life time), filament radius and length are all interdependent. The surface area of the filament can be maximized by increasing $r$. From Equation (7), when the power is increased, it is desirable to increase $r$ and $L$ to minimize the increase in temperature $T$. Equations (6) and (7) can be equated and we get

$$
T^{4}=\frac{V^{2} r}{2 \varepsilon \sigma \rho L^{2}}
$$

This equation shows the interdependence of filament temperature and filament radius and length. Tungsten filament emissivity directly affects relationship between surface temperature of a filament and its thermal radiation spectrum. 


\section{2 wavelength Calculation Method}

The wavelength of the emitted radiation has been calculated by using the following relation.

$$
\text { Wavelength }(\lambda)=\frac{h c}{4.965 k T}
$$

Where $h$ is the Planck's constant, $c$ is the velocity of light, $k$ is the Boltzmann's constant, and $T$ is the temperature of the body [11].

\subsection{Secondary Data Acquisition}

The values of length and diameter of filament bulbs shown in Table 5 had been measured by Agrawal D.C. He used these values to estimate the values of pitch ratio and mandrel ratio [18]. These values of length and diameter of the filaments were measured by using scanning electron microscope (SEM) image [18].

Table 5: Different wattage bulb with their filament length and diameter $\{$ Ref: [18]\}

\begin{tabular}{lll}
\hline Lamp watt $P$ & Length $L$ cm & Diameter $D$ cm \\
\hline 6 & 37.084 & 0.001143 \\
25 & 56.388 & 0.003048 \\
60 & 53.340 & 0.004572 \\
100 & 57.912 & 0.006350 \\
300 & 72.390 & 0.012700 \\
500 & 87.376 & 0.018034 \\
\hline
\end{tabular}

\section{RESULT AND DISCUSSION}

The different values of radiant emittance, radiance, peak spectral radiance, spectral radiance and band radiance are calculated for different wattage bulbs. These values are obtained by using Hi-Resolution spectral calculator [19]. On keeping different values of emissivity, the same wattage bulb also has different temperatures even the value of resistivity is taken constant. The lower and upper limit of the wavelength is taken as $0.27 \mu \mathrm{m}$ and $6 \mu \mathrm{m}$ respectively. In the Figures 1-6, the $y$-axis represents the amount of energy radiated by a tungsten filament at a specific wavelength while the $\mathrm{x}$-axis represents the wavelength. By locating the peak (highest point) of the curve, it can be determined, "at what wavelength the greatest amount of energy is emitted at".

\section{1 evaluation of Temperature}

Temperature and peak emission wavelength are calculated by using Equation 8 and Equation 9 respectively. The calculated values of temperatures (with different values of emissivity) and peak emission wavelengths for 6,60 , and 500 watt are shown in Table 6-8. The behavior of 6 watt, 60 watt and 500 watt bulb is discussed in section 4.2, 4.3, and 4.4 respectively.

\subsection{For 6 Watt Bulb}

Temperature, emissivity and peak emission wavelength for 6 watt bulb are shown in Table 6.The radiant emittance, radiance, peak spectral radiance for same wattage bulb but at different values of emissivity are shown in Table 6(a) and 6(b).

Table 6: Temperature, emissivity and peak emission wavelength profile for 6 watt bulb

\begin{tabular}{lll}
\hline Temperature $(T)($ in $K)$ & Emissivity $(\varepsilon)$ & $\begin{array}{l}\text { Peak emission wavelength }\left(\lambda_{\max }\right) \\
\text { (in meter) }\end{array}$ \\
\hline 2015 & 0.433 & $1.438 \times 10^{-6} \mathrm{~m}$ \\
2024 & 0.431 & $1.432 \times 10^{-6} \mathrm{~m}$ \\
2043 & 0.427 & $1.419 \times 10^{-6} \mathrm{~m}$ \\
2073 & 0.421 & $1.398 \times 10^{-6} \mathrm{~m}$ \\
2103 & 0.415 & $1.378 \times 10^{-6} \mathrm{~m}$ \\
\hline
\end{tabular}


Table 6(a): Radiant emittance, radiance, peak spectral radiance, spectral radiance, band radiance profile at temperature $2015 \mathrm{~K}$

\begin{tabular}{l|l}
\hline Inputs & Results \\
\hline Temperature: $2015 \mathrm{~K}$ & Radiant emittance: $404773 \mathrm{w} / \mathrm{m}^{2}$ \\
Emissivity: 0.433 & Radiance: $128843 \mathrm{w} / \mathrm{m}^{2} / \mathrm{sr}$ \\
Wavelength: $1.438 \times 10^{-6} \mathrm{~m}$ & Peak Spectral radiance: $58911.9 \mathrm{w} / \mathrm{m}^{2} / \mathrm{sr} / \mu \mathrm{m}$ \\
Lower limit: $2.7 \times 10^{-7} \mathrm{~m}$ & Wavelength of peak: $1.43809 \mu \mathrm{m}$ \\
Upper limit: $6 \times 10^{-6} \mathrm{~m}$ & Spectral Radiance: $58911.9 \mathrm{w} / \mathrm{m}^{2} / \mathrm{sr} / \mu \mathrm{m}$ \\
& $(7.23906 \mathrm{e}+18$ photons $/ \mathrm{J})$ \\
& Band Radiance: $121895 \mathrm{w} / \mathrm{m}^{2} / \mathrm{sr}$ \\
\hline
\end{tabular}

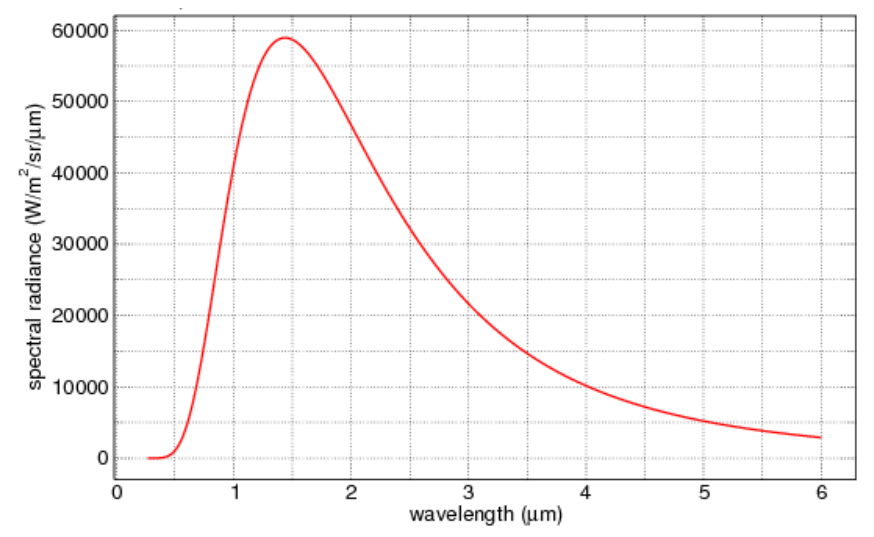

Fig. 1: Variation of spectral radiance with wavelength for temperature $2015 \mathrm{~K}$ and emissivity 0.433

Table 6(b): Radiant emittance, radiance, peak spectral radiance, spectral radiance, band radiance profile at temperature $2103 \mathrm{~K}$ and emissivity 0.415

\begin{tabular}{l|l}
\hline Inputs & Results \\
\hline Temperature: $2103 \mathrm{~K}$ & Radiant emittance: $460287 \mathrm{w} / \mathrm{m}^{2}$ \\
Emissivity: 0.415 & Radiance: $146514 \mathrm{w} / \mathrm{m}^{2} / \mathrm{sr}$ \\
Wavelength: $1.378 \times 10^{-6} \mathrm{~m}$ & Peak Spectral radiance: $69917.2 \mathrm{w} / \mathrm{m}^{2} / \mathrm{sr} / \mu \mathrm{m}$ \\
Lower limit: $2.7 \times 10^{-7} \mathrm{~m}$ & Wavelength of peak: $1.37791 \mu \mathrm{m}$ \\
Upper limit: $6 \times 10^{-6} \mathrm{~m}$ & Spectral Radiance: $69917.2 \mathrm{w} / \mathrm{m}^{2} / \mathrm{sr} / \mu \mathrm{m}$ \\
& $(6.93701 \mathrm{e}+18$ photons $/ \mathrm{J})$ \\
& Band Radiance: $139418 \mathrm{w} / \mathrm{m}^{2} / \mathrm{sr}$ \\
\hline
\end{tabular}

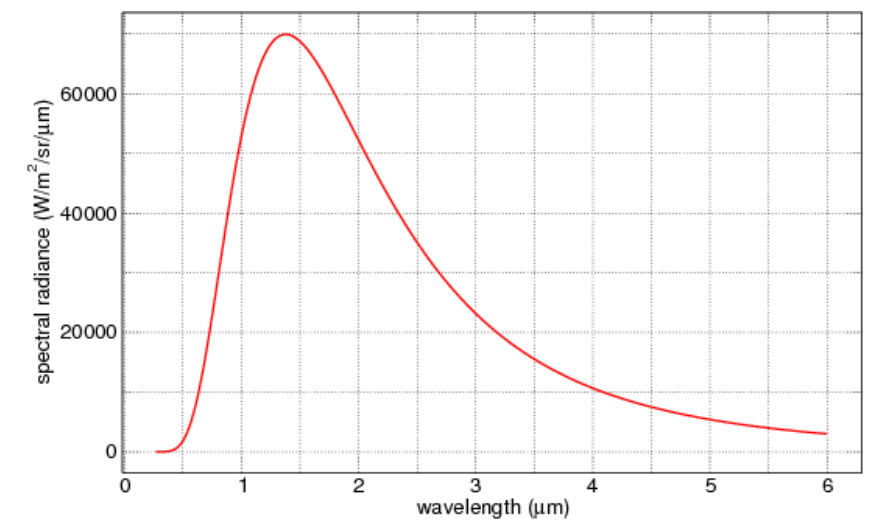

Fig. 2: Variation of spectral radiance with wavelength for temperature $2103 \mathrm{~K}$ and emissivity 0.415 
The number of radiations emitted from lower wattage bulb is greater than the number of emitted radiations from the higher wattage bulb as shown in Table 6(a) and 6(b). The energy emitted per unit area per unit time is greater for the filament which gets heated at a higher temperature. The temperature depends upon the emissivity. When the emissivity is 0.433 , then at a point on the surface of a source (filament), the radiant power emitted per unit area of source is 404773 but when the emissivity is 0.415 then at a point on the surface of the source, the radiant power emitted per unit area of the source is 460287. Also, for higher temperature $2103 \mathrm{~K}$ at lower emissivity 0.415 ( see Table 6(b)), the radiant power leaving an element of the surface at the point divided by the area of the element is greater than for lower temperature $2015 \mathrm{~K}$ at higher emissivity 0.433 (see Table 6(a)).

For same wattage bulb, the value of spectral radiance increases if emissivity decreases as shown in Fig. 1-2. The value of peak emission wavelength is directly proportional to the value of emissivity as shown in Table 6.

\subsection{For 60 Watt Bulb}

For 60 watt bulb, the values of temperature and peak emission wavelength obtained by using equation 8 and 9 respectively are given in Table 7 . The different values of emissivity shown in Table 7 are taken from Table 3.The value of length and diameter for the 60 watt tungsten bulb is given in Table 5.The value of resistivity is taken constant which is equal to $7.391 \times 10^{-5} \Omega \mathrm{cm}$.

Table 7: Temperature, emissivity and peak emission wavelength profile for 60 watt bulb

\begin{tabular}{lll}
\hline Temperature $(T)($ in $K)$ & Emissivity $(\varepsilon)$ & $\begin{array}{l}\text { Peak emission wavelength }\left(\lambda_{\max }\right)(\text { in } \\
\text { meter })\end{array}$ \\
\hline 2376 & 0.433 & $1.22 \times 10^{-6}$ \\
2387 & 0.431 & $1.214 \times 10^{-6}$ \\
2410 & 0.427 & $1.202 \times 10^{-6}$ \\
2444 & 0.421 & $1.186 \times 10^{-6}$ \\
2479 & 0.415 & $1.169 \times 10^{-6}$ \\
\hline
\end{tabular}

Table 7(a): Radiant emittance, radiance, peak spectral radiance, spectral radiance, band radiance profile at temperature $2376 \mathrm{~K}$ and emissivity 0.433

\begin{tabular}{l|l}
\hline Inputs & Results \\
\hline Temperature: $2376 \mathrm{~K}$ & Radiant emittance: $782523 \mathrm{w} / \mathrm{m}^{2}$ \\
Emissivity: 0.433 & Radiance: $249085 \mathrm{w} / \mathrm{m}^{2} / \mathrm{sr}$ \\
Wavelength: $1.22 \times 10^{-6} \mathrm{~m}$ & Peak Spectral radiance: $134295 \mathrm{w} / \mathrm{m}^{2} / \mathrm{sr} / \mu \mathrm{m}$ \\
Lower limit: $2.7 \times 10^{-7} \mathrm{~m}$ & Wavelength of peak: $1.21959 \mu \mathrm{m}$ \\
Upper limit: $6 \times 10^{-6} \mathrm{~m}$ & Spectral Radiance: $134295 \mathrm{w} / \mathrm{m}^{2} / \mathrm{sr} / \mu \mathrm{m}$ \\
& $(6.14162 \mathrm{e}+18$ photons $/ \mathrm{J})$ \\
& Band Radiance: $240254 \mathrm{w} / \mathrm{m}^{2} / \mathrm{sr}$ \\
\hline
\end{tabular}




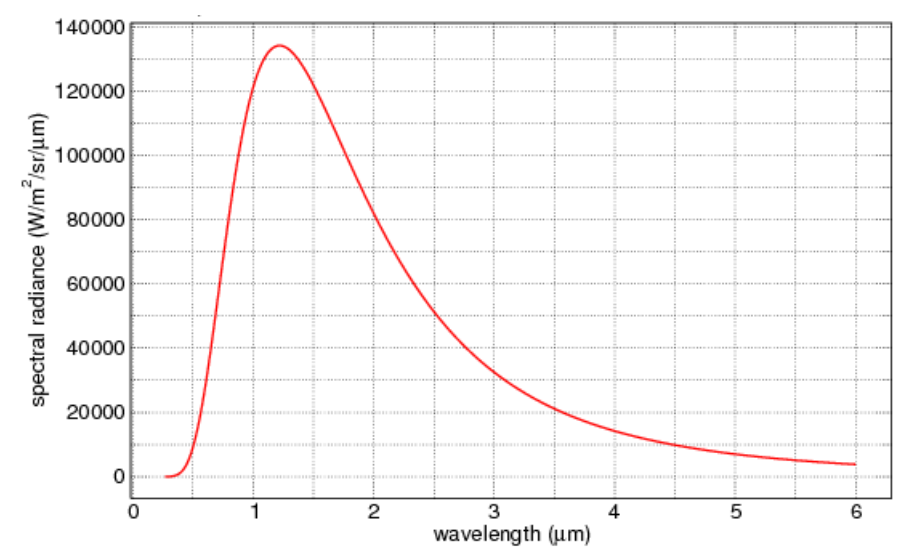

Fig. 3: Variation of spectral radiance with wavelength for temperature $2376 \mathrm{~K}$ and emissivity 0.433

Table 7(b): Radiant emittance, radiance, peak spectral radiance, spectral radiance, band radiance profile at temperature $2479 \mathrm{~K}$ and emissivity 0.415

\begin{tabular}{l|l}
\hline Inputs & Results \\
\hline Temperature: $2479 \mathrm{~K}$ & Radiant emittance: $888746 \mathrm{w} / \mathrm{m}^{2}$ \\
Emissivity: 0.415 & Radiance: $282896 \mathrm{w} / \mathrm{m}^{2} / \mathrm{sr}$ \\
Wavelength: $1.169 \times 10^{-6} \mathrm{~m}$ & Peak Spectral radiance: $159137 \mathrm{w} / \mathrm{m}^{2} / \mathrm{sr} / \mu \mathrm{m}$ \\
Lower limit: $2.7 \times 10^{-7} \mathrm{~m}$ & Wavelength of peak: $1.16892 \mu \mathrm{m}$ \\
Upper limit: $6 \times 10^{-6} \mathrm{~m}$ & Spectral Radiance: $159137 \mathrm{w} / \mathrm{m}^{2} / \mathrm{sr} / \mu \mathrm{m}$ \\
& $(5.88488 \mathrm{e}+18$ photons $/ \mathrm{J})$ \\
& Band Radiance: $273913 \mathrm{w} / \mathrm{m}^{2} / \mathrm{sr}$ \\
\hline
\end{tabular}

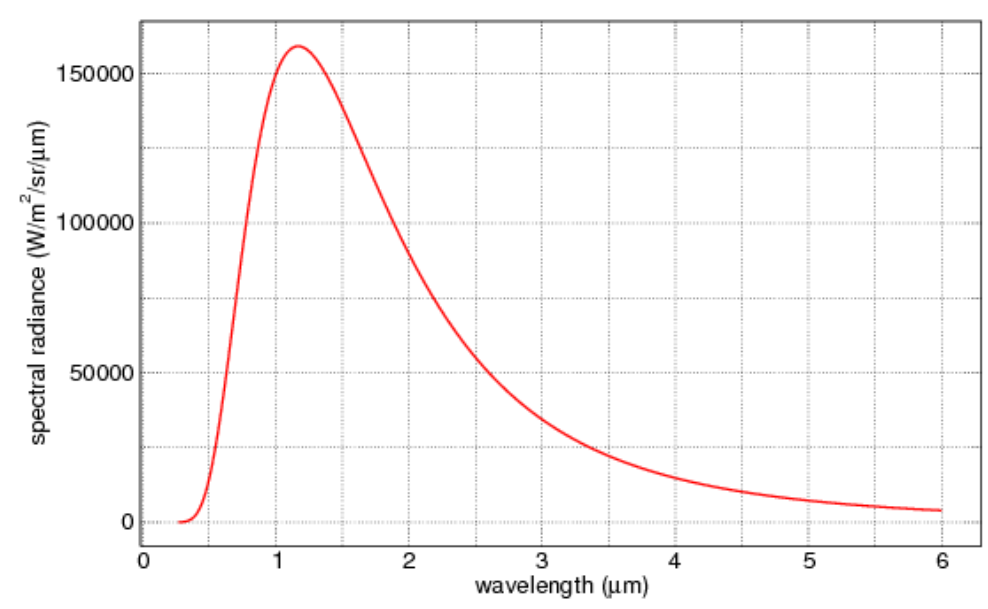

Fig. 4: Variation of spectral radiance with wavelength for temperature $2479 \mathrm{~K}$ and emissivity 0.415

The value of radiant emittance, band radiance, and spectral radiance is greater for emissivity 0.415 , than for emissivity 0.433 which is shown in Table 7(a) and 7(b). The area under the curve is greater for $2376 \mathrm{~K}$ filament temperature than the $2479 \mathrm{~K}$ filament temperature which is shown in Fig. 3-4. The number of emitted radiations is maximum for lower temperature filament and minimum for higher temperature filament.

\subsection{For 500 Watt Bulb}

For 500 watt bulb, the temperature and peak emission wavelength are calculated by using Equation 8 and Equations 9 respectively. The length and diameter for 500 watt filament bulb are given in Table 5 . 
Table 8: Temperature, emissivity, and peak emission wavelength profile for 500 watt bulb

\begin{tabular}{lll}
\hline Temperature $(T)($ in $K)$ & Emissivity $(\varepsilon)$ & Peak emission wavelength $\left(\lambda_{\max }\right)$ (in meter) \\
\hline 2616 & 0.433 & $1.108 \times 10^{-6}$ \\
2629 & 0.431 & $1.102 \times 10^{-6}$ \\
2653 & 0.427 & $1.092 \times 10^{-6}$ \\
2691 & 0.421 & $1.077 \times 10^{-6}$ \\
2730 & 0.415 & $1.061 \times 10^{-6}$ \\
\hline
\end{tabular}

Table 8(a): Radiant emittance, radiance, peak spectral radiance, spectral radiance, band radiance profile at temperature $2616 \mathrm{~K}$ and emissivity 0.433

\begin{tabular}{l|l}
\hline Inputs & Results \\
\hline Temperature: $2616 \mathrm{~K}$ & Radiant emittance: $1.14991 \mathrm{e}+06 \mathrm{w} / \mathrm{m}^{2}$ \\
Emissivity: 0.433 & Radiance: $366026 \mathrm{w} / \mathrm{m}^{2} / \mathrm{sr}$ \\
Wavelength: $1.108 \times 10^{-6} \mathrm{~m}$ & Peak Spectral radiance: $217278 \mathrm{w} / \mathrm{m}^{2} / \mathrm{sr} / \mu \mathrm{m}$ \\
Lower limit: $2.7 \times 10^{-7} \mathrm{~m}$ & Wavelength of peak: $1.1077 \mu \mathrm{m}$ \\
Upper limit: $6 \times 10^{-6} \mathrm{~m}$ & Spectral Radiance: $217278 \mathrm{w} / \mathrm{m}^{2} / \mathrm{sr} / \mu \mathrm{m}$ \\
& $(5.5778 \mathrm{e}+18$ photons $/ \mathrm{J})$ \\
& Band Radiance: $355927 \mathrm{w} / \mathrm{m}^{2} / \mathrm{sr}$ \\
\hline
\end{tabular}

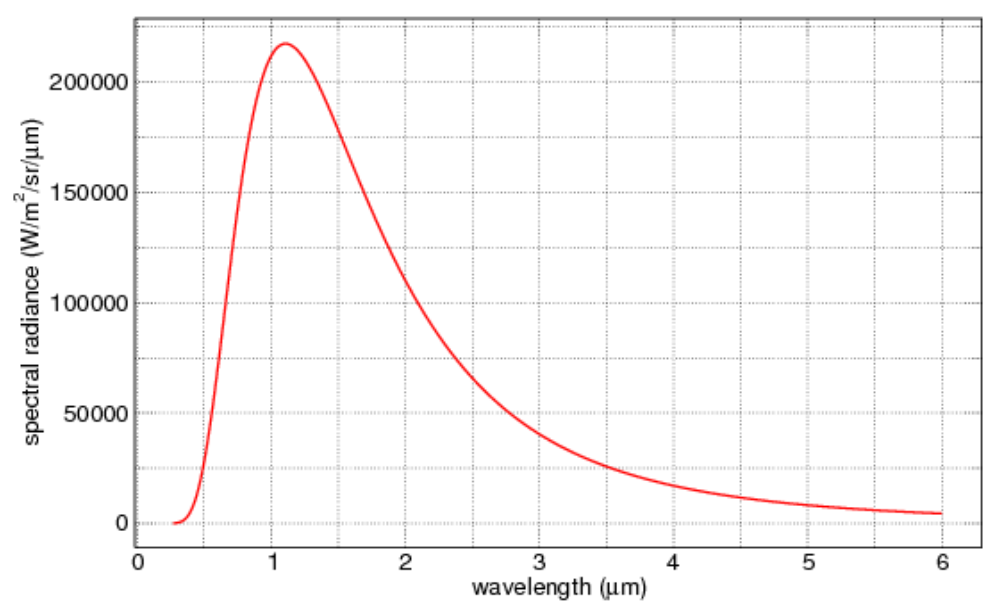

Fig. 5: Variation of spectral radiance with wavelength for temperature $2616 \mathrm{~K}$ and emissivity 0.433

Table 8(b): Radiant emittance, radiance, peak spectral radiance, spectral radiance, band radiance profile at temperature $2730 \mathrm{~K}$ and emissivity 0.415 .

\begin{tabular}{l|l}
\hline Inputs & Results \\
\hline Temperature: $2730 \mathrm{~K}$ & Radiant emittance: $1.30714 \mathrm{e}+06 \mathrm{w} / \mathrm{m}^{2}$ \\
Emissivity: 0.415 & Radiance: $416075 \mathrm{w} / \mathrm{m}^{2} / \mathrm{sr}$ \\
Wavelength: $1.061 \times 10^{-6} \mathrm{~m}$ & Peak Spectral radiance: $257751 \mathrm{w} / \mathrm{m}^{2} / \mathrm{sr} / \mu \mathrm{m}$ \\
Lower limit: $2.7 \times 10^{-7} \mathrm{~m}$ & Wavelength of peak: $1.06145 \mu \mathrm{m}$ \\
Upper limit: $6 \times 10^{-6} \mathrm{~m}$ & Spectral Radiance: $257751 \mathrm{w} / \mathrm{m}^{2} / \mathrm{sr} / \mu \mathrm{m}$ \\
& $(5.3412 \mathrm{e}+18$ photons $/ \mathrm{J})$ \\
\hline
\end{tabular}




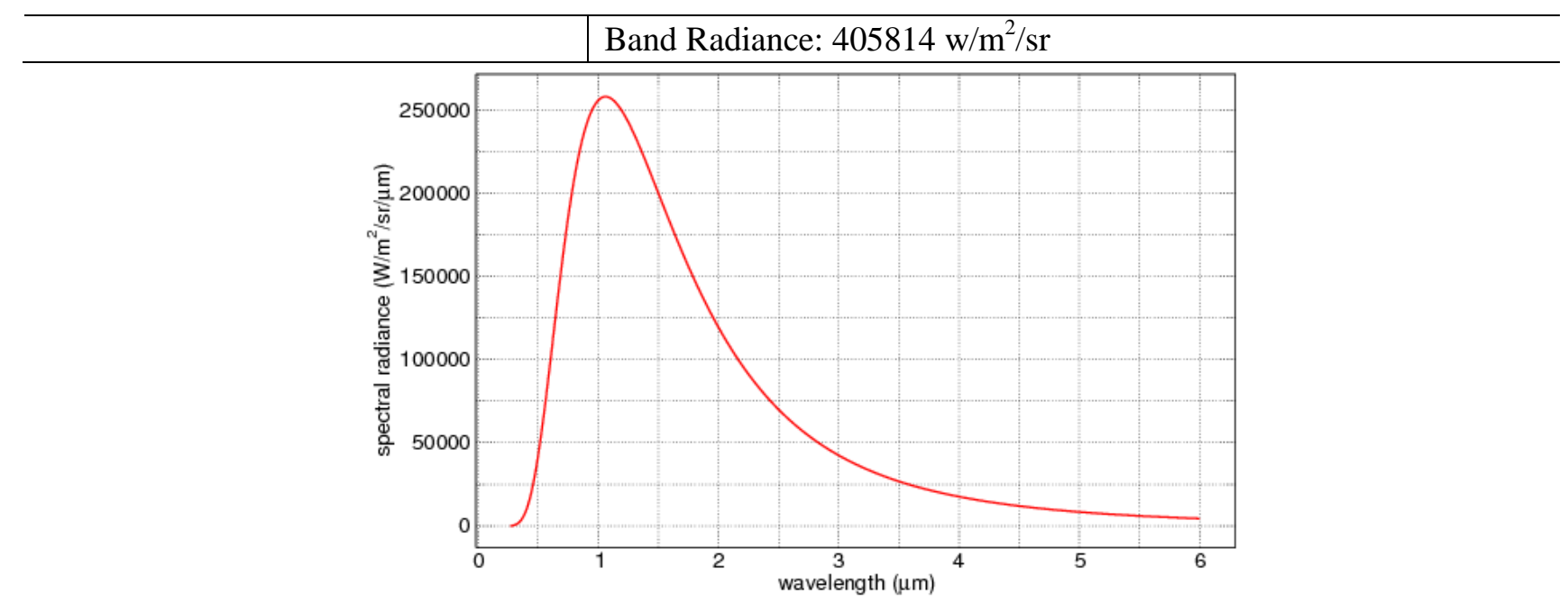

Fig. 6: Variation of spectral radiance with wavelength for temperature $2730 \mathrm{~K}$ and emissivity 0.415

The value of radiant emittance, radiance, spectral radiance and band radiance increases if temperature increases which is shown in Fig. 1-6. The spectral radiance, radiant emittance, does not depend only on emissivity but also on temperature.

The radiance and radiant emittance differs only by a small value for same wattage bulb, independent on emissivity of the filament. For lower value of emissivity, the number of emitted radiations is few. More radiations are emitted when the emissivity is higher. For the higher temperature tungsten, the radiant power leaving an element of the source at the point is greater than that of the lower temperature tungsten which is shown in Table 6-8. Intensity of the radiations from lower emissivity bulb is less than that of higher emissivity bulb. More lower the value of emissivity, narrower the peak of the emission curve. If the value of emissivity goes on increasing, the peak of the emission curve becomes more and more flattened. The more flattened peak of the emission curve indicates presence of more infrared radiations. The narrower peak of the curve indicates presence of few amounts of low energy radiations in comparison to the blackbody emission curve having flattened peak. On increasing emissivity, wavelength of peak also increases as shown in Fig. 1-6. The probability distribution of the emitted radiations is shown in Fig. 1-6.

If the constant value (4.965) in the denominator is made equal to one, the value of peak emission wavelength decreases. If the value of peak emission wavelength is shorter, the probability distribution of the emitted radiations is such that there exists maximum amount of the emitted radiations which fall in the shorter wavelength (shifts towards the shorter ultraviolet radiations). Similarly, if the constant value (4.965) in the denominator of the right side of the Wien's displacement law is lowered, the value of peak emission wavelength increases. If the value of the peak emission wavelength is greater, the probability distribution of the emitted radiations is such that there exists maximum amount of the longer wavelength radiations and very few amounts of visible radiations whose intensity is not sufficient to the eyes to make the object visible. So the equation $\left(\lambda_{\max }\right)=\frac{h c}{4.965 k T}$ can be used to analyze the electromagnetic spectrum emitted from the filament bulb. Fig. from 1-6 tells that the value of emissivity must lie in the intermediate level for the filament as shown in Table 1.

At $\varepsilon \rightarrow 0, T \rightarrow \infty$. If temperature tends to infinity, then from Wien's displacement law peak emission wavelength is infinitely small. At smaller value of peak emission wavelength, the distribution of radiations is such that they almost fall towards smaller wavelength region, which is against the nature of the radiations emitted from the filament bulb. For same wattage bulb, on lowering the value of emissivity, the amount of emitted lower wavelength radiations goes on decreasing. The number of radiations emitted from lower wattage bulb is greater than the number of emitted radiations from the higher wattage bulb as shown in Fig. from 1-6. The emitted radiations which have longer wavelength are more intense and the emitted radiations which have shorter wavelength are less intense. 
The area under the blackbody spectrum curve contains very few longer and shorter wavelength radiations and intermediate amount of visible and infrared radiations. The curve slightly goes on shifting towards the left as shown in Fig. 1-6 which indicates the increase in comparatively shorter wavelength as temperature increases. The curve towards the right about the peak becomes steeper if the temperature goes on increasing (see Fig. 1-6). The value of peak emission wavelength obtained by using equation 9 which has been used to determine the probability distribution of the spectrum of the wavelength of the emitted radiations justifies the modality that have been assumed for the work.

\section{CONCLUSION}

The Wien's displacement law can be used for a body, which is not perfectly black. Wien's displacement equation can be used for a radiation emitting body, which comprises both shorter and longer wavelength. Tungsten filament (blackbody) emits radiation across multiple wavelength at which most of the radiation will be emitted. The cooler the filament, the longer, the wavelength at which most of the radiations will be emitted. Conversely, the hotter the filament, the shorter the wavelength at which the peak radiation is emitted. At lower filament temperature, spectrum contains maximum number of lower energy range radiations and comparatively minimum number of higher energy range radiations. The lower wattage bulb gets heated at a lower temperature and higher wattage bulb gets heated at a higher temperature. So the total energy of the emitted radiations also goes on increasing with increase in power (temperature) of the bulb. So, the bulb of higher wattage gives more visibility than lower wattage bulb.

The peak emission wavelength which depends upon the temperatures of the filament is used to know "how the spectrum of the wavelength of the emitted radiations is distributed". Temperature, emissivity and peak emission wavelength are all interdependence. From this it can be concluded that the intensity of the emitted radiations is inversely proportional to their wavelength. The energy emitted per unit area per unit time is greater for higher wattage bulb and lesser for lower wattage bulb. The black body emission curve shows that maximum amount of infrared radiations are emitted from the filament bulb and almost absence of ultraviolet radiations. All results shown in both table (see Table 6-8) and figures (see Fig. 1-6) are obtained by the use of Wien's displacement equation. The result obtained in Table 6-8 is shown in Fig. 1- 6 agrees with the modality that has been made for whole work. This confirms the validity of Wien's displacement law on Tungsten bulb.

\section{REFERENCES}

1. Jones, H. A. The Characteristics of Tungsten Filaments as Functions of Temperature. General Electric Review, 30: 310 (1927).

2. Carla Isabel, R. Starlight inside a Light Bulb. The European Journal for Science Teachers, 31: 2 (2015).

3. Ranganath, G.S. Blackbody Radiation. Resonance, 13: 115-133 (2008).

4. Yang, X.; Wei, B. Exact Research on the Theory of the Blackbody Thermal Radiation. Sci Rep 6: 37214, 1-3 (2016).

5. David, H. S.; Maurice, B. and William, M. Infrared, Visible, and Ultraviolet Radiation. John Wiley \& Sons, Hoboken (2012).

6. Ling, S. J. The Electromagnetic Spectrum. University Physics. 2: 364 (2016).

7. Pidwirny, M. The Nature of Radiation. In: Fundamentals of Physical Geography, 2nd Edition, University of British Columbia, Okanagan, 115 (2006).

8. Andrew Zimmerman, J. Understanding the Colors That Make up White Light (2020). https://www.thoughtco.com/the visible-lightspectrum-2699036

9. Rossow, R. A. Blackbody Temperature Calculations from Visible and Near-IR Spectra for Gas-Fired Furnaces. The Faculty of the Graduate School, University of Missouri, Columbia, 34-35 (2005).

10. Riedl, M. Optical Design Fundamentals for Infrared Systems, Second Edition, SPIE Press, Bellingham, WA (2001).

11. Singhal, S. S.; Agarwal, J. P. and Satya, P. Heat Thermodynamics and Statistical Physics. Pragati Prakashan, Meerut, 379-380 (1998).

12. David, W. Bell. Wien's Displacement law as a function of frequency. Journal of Chemical Education, 90(9): 1250-1252 (2013).

13. Ralph, A. F. Tungsten Filament Emissivity Behavior. FAR Associates 1532, Newport Drive, Macedonia, 1-6 (2006).

14. Zhao, L.; Li, H. W.; Ning, W.; Shi, Q.; Jose, A.; S., Zhengming, W.; Bo-Hui, T.; and Guangjian, Y. Land Surface Emissivity Retrieval from Satellite Data. International Journal of Remote Sensing, 34: 3084-3127 (2013). 
15. Forsythe, W. E. and Worthing, A. G. The Properties of Tungsten and the Characteristics of the Tungsten Lamps. Astrophysical Journal, 61: 146 (1925).

16. Blevin, W. R. and Brown, W. J. A Precise Measurement of the Stefan-Boltzmann Constant. Metrologia, 7: 15 (1971).

17. Pathare, S. R.; Lahane, R. D.; Sawant, S. S. and Patil, C. C. Power Loss from Hot Tungsten Filament. 8 (2010).

18. Agrawal, D. C. The Coiling Factor in the Tungsten Filament Lamps. Latin American Journal of Physics Education, 5: 443-449 (2011).

19. Mitchell, B.; Ekey, R.; McCullough, R. and Reitz, W. A Fantastic Quantitave Exploration of Ohm's Law. The Physics Teacher, 56: 75-78 (2018).

20. Carla, M. Stefan-Boltzmann Law for the Tungsten Filament of a Light Bulb: Revisiting the Experiment. American Journal of Physics, 81: 512-517 (2013). 